\title{
Fuerzas Ortodóncicas como Agentes Vulnerantes de la Salud Pulpar. Reporte de Dos Casos
}

\author{
Orthodontic Forces Causing Damage the Pulpal Condition. Report of Two Cases
}

Gabriel M. Fonseca \& Andrés E. Guzmán

FONSECA, G. M. \& GUZMÁN, A. E. Fuerzas ortodóncicas como agentes vulnerantes de la salud pulpar. Reporte de dos casos. Int. J. Odontostomat., 4(3):271-276, 2010.

RESUMEN: La literatura ya ha sugerido la implicancia del uso de fuerzas ortodóncicas como productoras de daño mecánico, reacción inflamatoria periodontal, lesión periapical y reabsorción radicular. Los parámetros más comúnmente explorados en investigaciones de la respuesta tisular a fuerzas ortodónticas consisten en medir los niveles de oxígeno pulpar, la vasculatura y los cambios en la circulación sanguínea. Se ha demostrado que la irrigación pulpar disminuye tempranamente cuando se realizan aplicaciones continuas de fuerzas ortodóncicas, aun si éstas son de intensidad suave, concluyendo que la magnitud de las fuerzas no necesariamente debe ser excesiva para originar daño celular, el que eventualmente podría derivar en cuadros que van desde simples cambios de color coronario hasta obliteraciones camerales o la misma necrosis pulpar. Se presentan dos casos de pacientes tratados ortodóncicamente con extravasación hemorrágica y necrosis pulpar (canino e incisivo lateral superiores izquierdos respectivamente), con ausencia informada de traumatismo previo, y se discuten protocolos diagnósticos para la evaluación de la salud pulpar.

PALABRAS CLAVE: ortodoncia, lesión pulpar, procedimientos diagnósticos.

\section{INTRODUCCIÓN}

Las fuerzas ortodóncicas son conocidas por producir daño mecánico y reacciones inflamatorias periodontales, lesión periapical y reabsorción radicular (Atack, 1999; Brezniak \& Wasserstein, 2001), así como también daño celular, cambios inflamatorios y alteraciones circulatorias en la pulpa dental (Mostafa et al., 1991; Nixon et al., 1993; Yamaguchi \& Kasai, 2005). Diferentes estudios han informado que estas fuerzas producen una reducción en los niveles de oxígeno, isquemia e hipoxia, dentro de las células pulpares (Derringer et al., 1996; Lau \& Wong, 2006).

Los parámetros más comúnmente explorados en investigaciones de la respuesta tisular a fuerzas ortodóncicas consisten en medir la vasculatura pulpar y los cambios en la circulación sanguínea (Santamaria et al., 2006). McDonald \& Pitt Ford (1994), utilizando láser doppler, encontraron que la irrigación pulpar humana disminuye ya a los 32 minutos de realizadas aplicaciones continuas de fuerzas suaves ortodóncicas.
Se ha referido que pequeñas fuerzas, incluso de corta duración, pueden originar respuestas celulares (Roberts \& Ferguson, 1989).

Estudios recientes han revelado que neuropéptidos como el relacionado con el gen de la calcitonina (calcitonine gene-related peptide -CGRP) y la sustancia $P(S P)$ pueden verse aumentados en la pulpa dental como resultado de la aplicación de fuerzas ortodóncicas, lo que se ha informado como claro indicador de mediación inflamatoria (Yamaguchi \& Kasai). Si bien se ha referido que algunas de estas pulpitis son usualmente transitorias, no significativas a largo plazo, y que la pérdida de la vitalidad pulpar por esta causa es una situación de rara frecuencia (Spector et al., 1974; Lau \& Wong), se ha informado que dientes traumatizados previamente aumentan sus posibilidades de mortificación al ser sometidos a tratamiento ortodóncico (Bauss et al., 2009; Bauss et al., 2010). Así mismo, los reportes de necrosis pulpares 
posteriores a tratamiento ortodóncico en dientes no traumatizados son escasos (Spector et al.; Popp et al., 1992), y si bien se ha explicado esta situación por variaciones anatómicas de los forámenes apicales o anomalías vasculares, se ha sugerido el no descartar la posibilidad de traumatismo previo no informado (Bauss et al., 2010).

Se presentan dos casos de pacientes tratados ortodóncicamente (canino e incisivo lateral superiores izquierdos), con ausencia informada de traumatismo previo, y la probable relación con extravasación hemorrágica y necrosis pulpares, y se discuten protocolos diagnósticos para la evaluación de la salud pulpar.

\section{CASO 1}

Paciente de 40 años con canino superior izquierdo presentando coloración coronaria amarronada
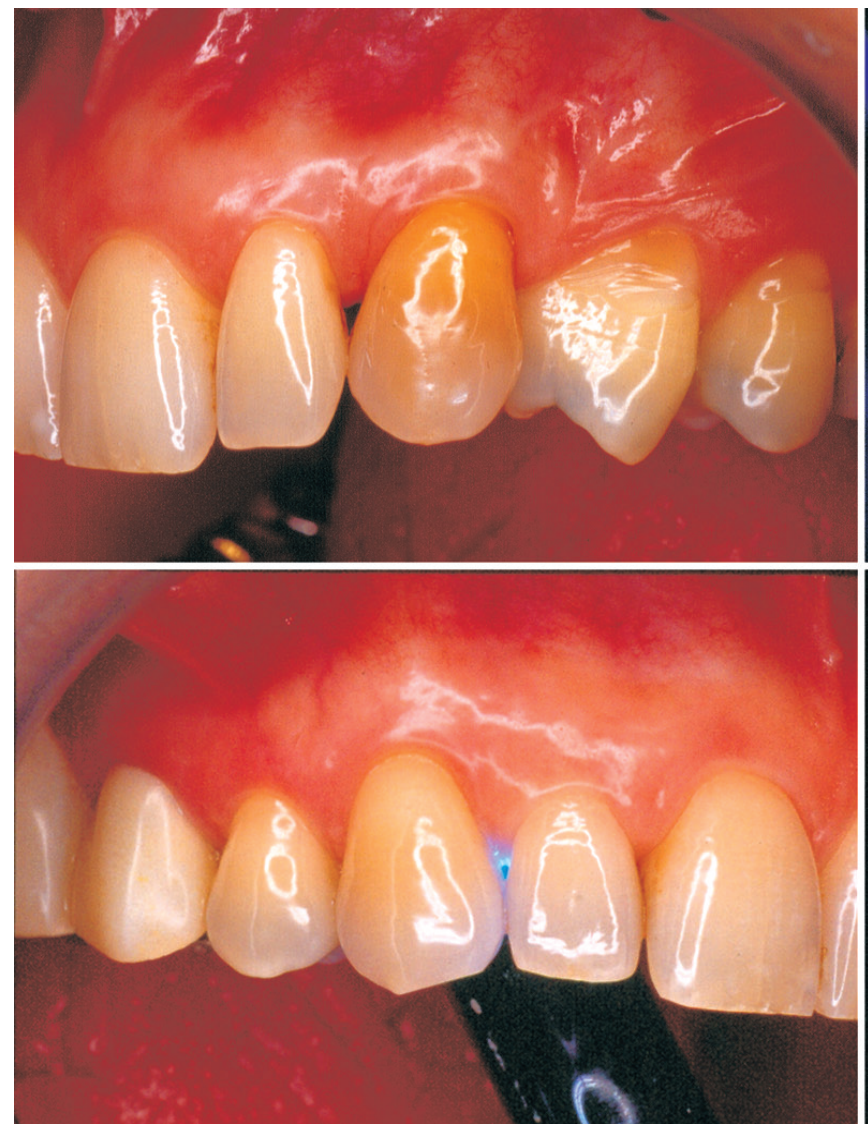

Fig. 1. Arriba: Canino superior izquierdo con tonalidad coronaria amarillenta amarronada. Obsérvese la opacidad localizada evidente en la maniobra de transiluminación (derecha), orientativa al diagnóstico de extravasación hemorrágica. Abajo: Canino superior derecho con características normales y traslucidez homogénea a la transiluminación (Lámpara de luz halógena Ventura ${ }^{\mathrm{TM}}$ modelo multilux/lux - serie 5986 y Unidad de fotocurado Ultrablue IS - KAVO ${ }^{\mathrm{TM}}$ alimentada por conversor de energía. y signos de vitalidad positiva alterada a las pruebas térmicas (reacción disminuida al frío y negativa al calor). La técnica de transiluminación, reveló modificación estructural con pérdida de la translucidez en forma localizada, lo que orientó al diagnóstico de extravasación hemorrágica con pulpa vital (Fig. 1). La radiografía periapical no evidenció de signos de anormalidad (Fig. 2). La anamnesis refirió una posición original anómala con potencial retención, razones por la que se efectuó reposicionamiento con tunelización y traccionamiento ortodóncico a la edad de 18 años. Este tratamiento duró aproximadamente un año y medio, con intensos dolores en cada activación semanal de la tracción. Una vez realizado el actual diagnóstico, se indicó conducta expectante y controles de la vitalidad cada 6 meses sin cambios a la fecha.

\section{CASO 2}

Paciente de 19 años derivada para extracción dentaria por importante área radiolúcida en apical del
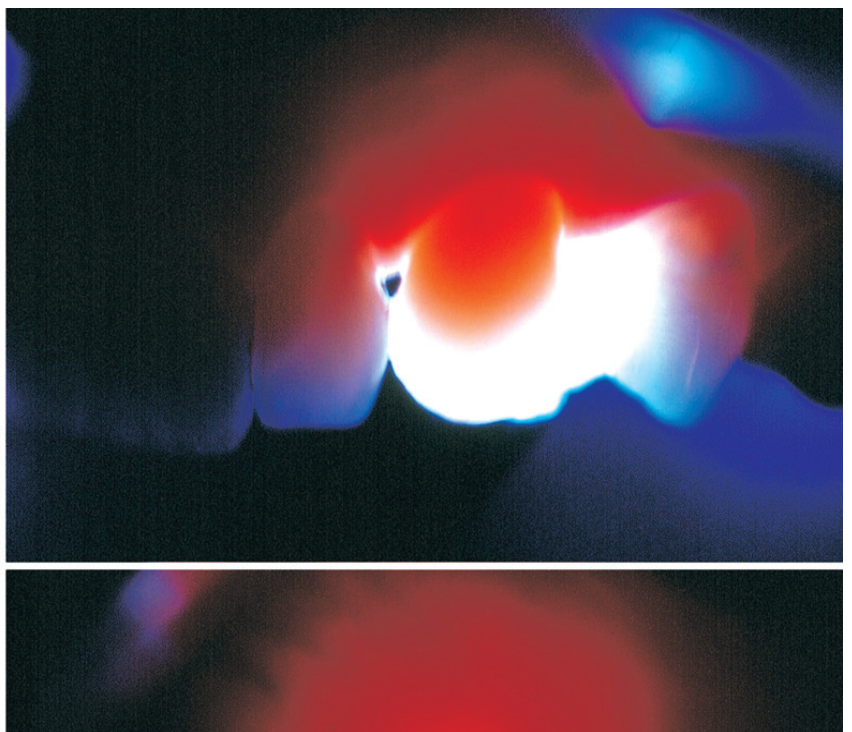


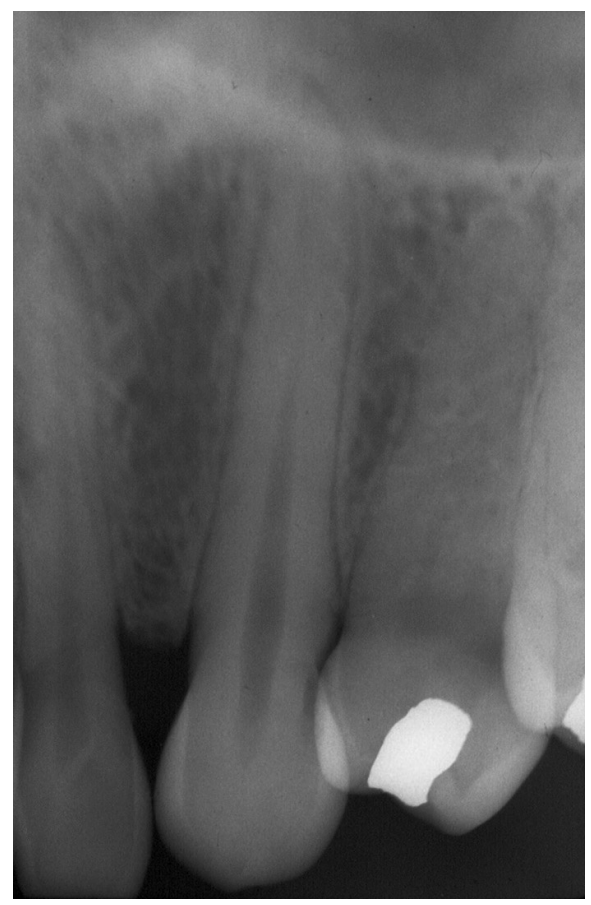

Fig. 2. Radiografía periapical, canino superior izquierdo. No se observan signos de anormalidad radiográfica.

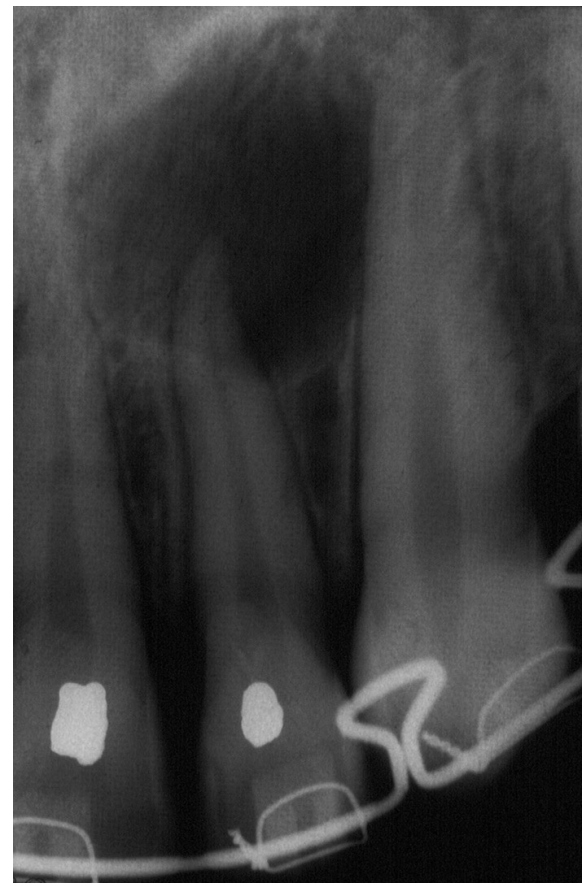

Fig. 3. Radiografía periapical, incisivo lateral superior izquierdo. Obsérvese importante área radiolúcida hacia distoapical del elemento problema. El profesional ortodoncista tratante, derivaba a la paciente para la extracción de dicho elemento ante un diagnóstico fallido por no realizar las pruebas de vitalidad pertinentes.

incisivo lateral superior izquierdo (Fig. 3). Si bien no se observaron evidencias del probable agente causal ni sintomatología dolorosa, la paciente refirió importante dolor inmediato a la colocación de los bracketts en ese diente durante una semana al momento de iniciar su tratamiento ortodóncico un año atrás. El diente problema, originalmente en vestibularización y sin traumatismo previo (según recordó la paciente), alcanzó la posición pretendida en solo dos meses. Las pruebas térmicas de vitalidad (omitidas por el profesional que efectuó la derivación), revelaron todas respuestas negativas con lo que se confirmó el diagnóstico de necrosis pulpar y se indicó su tratamiento endodóntico. Se corroboró la desaparición casi absoluta del proceso un año después (Fig. 4).

\section{DISCUSIÓN}

Lau \& Wong refieren que algunos grados de lesión pulpar son esperables en relación a la terapéutica ortodóncica, pero que estos son usualmente reversibles y no significativos a largo plazo. Los mismos Derringer et al., amparándose en los fenómenos de reparación y neovascularización pulpar y periodontal posteriores a la terapéutica ortodóncica, explican como "sorprendente" que aun con la aplicación de grandes fuerzas y desplazamientos de los dientes tratados a grandes distancias, estos tratamientos no originarían efectos iatrogénicos.

Sin embargo, Dhopatkar et al. (2005), sobre un modelo in vivo

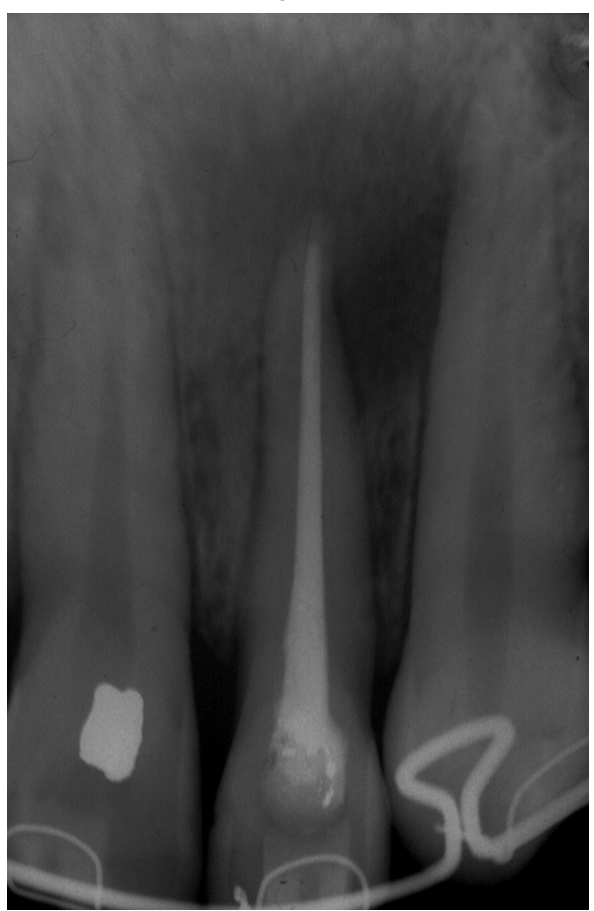

Fig. 4. Radiografía periapical, incisivo lateral superior izquierdo. Obsérvese la casi total desaparición del proceso radiolúcido a un año de realizado el tratamiento endodóntico. de ratas Wistar, han referido que el complejo dentino pulpar es altamente susceptible a la aplicación de fuerzas externas, que éstas pueden producir respuesta celular pulpar, independientemente de su naturaleza (compresión o tensión), y que incluso la expresión genética dentro del complejo dentino pulpar (en particular, genes asociados con la proliferación celular PCNA-) puede verse alterada. Sin lugar a dudas, la presencia de dolor (entendido éste como signo cardinal y asociación clara con la interacción de mediadores químicos en la inflamación) podría entrever un eventual daño pulpar, independientemente del tipo de esfuerzo aplicado y la posibilidad de recuperación (Yamaguchi \& Kasai). 
Se ha hecho una específica diferencia entre las respuestas pulpares de dientes traumatizados y no traumatizados en forma previa al tratamiento ortodóncico: Bauss et al. (2010) refieren que el sistema circulatorio pulpar en dientes no traumatizados parece poder adaptarse y compensar la reducción en la circulación sanguínea, mientras que en los traumatizados, la capacidad venosa resultaría insuficiente para mantener esta irrigación resultando en necrosis pulpar. Sin embargo, y aun cuando son pocos en comparación con el número de pacientes que reciben diariamente un tratamiento ortodóncico (Lau \& Wong), los casos de morbilidad o mortalidad de la pulpa hacen necesario un refinamiento en los procedimientos diagnósticos de su salud, como así también establecer rigurosos y regulares controles (Bauss et al., 2010) dado que no es sencillo definir cuáles son los niveles apropiados de fuerzas a aplicar entendiendo la gran variabilidad clínica determinada por las reacciones específicas individuales, el área de superficie radicular de la pieza involucrada o la misma pérdida de fricción dentro del tratamiento (Drenker, 1988; Derringer et al.).

Un estado pulpar alterado puede manifestarse mediante la detección de signos clínicos y/o radiológicos (Fonseca, 2009; Bauss et al., 2010). Los cambios de color coronario, si bien han sido por largo sugerentes de necrosis pulpar (Grossman, 1973), pueden también obedecer al efecto secundario de una extravasación hemorrágica o una metamorfosis calcificante, ambas patologías no necesariamente acompañadas por pulpas necróticas (Arwill, 1967; Amir et al., 2001; Rotstein, 2002; Sulieman, 2005; Fonseca; Bauss et al., 2010). Del mismo modo, la falta de respuesta sensible a las pruebas térmicas de vitalidad, no pueden por sí solas establecer un estado de necrosis dado el amplio rango de falsos positivo y negativo que pueden presentar (Fonseca). Solo la presencia de radiolucidez periapical puede ser considerada condición segura para diagnosticar un estado de muerte pulpar (Andreasen \& Andreasen, 2007; Bauss et al., 2010). Por otra parte, la transiluminación, procedimiento ampliamente difundido en la práctica diaria odontológica para la detección de microfracturas y alteraciones estructurales (Andreasen \& Andreasen, 2005) permitió en el primero de los casos, la detección de opacidad localizada asociada a la presencia de hemosiderina dentinaria consecuente con una extravasación hemorrágica distante cronológicamente.

En 1994, Woloshyn et al., sobre 32 pacientes tratados ortodóncicamente por retención de caninos superiores, seis casos (21\%) presentaron obliteración pulpar, 1 caso $(3,13 \%)$ evolucionó a necrosis, 8 casos (25\%) evidenciaron respuesta negativa a pruebas de vitalidad y 14 casos (45\%) presentaron cambio en el color de sus coronas. En los casos que se presentan, mientras que el primero de ellos pone en evidencia una manifestación tardía de morbilidad pulpar consecuente con un tratamiento ortodóncico de franca agresión mecánica, hoy solo manifiesto por un intenso cambio de coloración y disminución en la capacidad de reacción a las pruebas térmicas, el segundo ofrece un cuadro potencialmente iatrogénico de no ser efectuados los necesarios procedimientos diagnósticos: la desvinculación de la probable causa (tratamiento ortodóncico con fuerzas continuas) con losefectos (necrosis pulpar) y la no realización de las pruebas de vitalidad correspondientes por parte del profesional tratante, concluyó no solo en un claro error diagnóstico sino también en un eventual y grosero sesgo: el profesional tratante derivaba ese incisivo lateral para su extracción.

Se deben profundizar los mecanismos biológicos con la clínica de los movimientos ortodóncicos para obtener resultados más eficientes e inocuos para los tejidos dentoperiodontales. La aplicación de fuerzas ortodóncicas puede generar daño pulpar en diferentes grados, pero de manera similar al producido por un traumatismo agudo, por lo que deben discutirse las implicancias de estos procedimientos como potenciales eventos iatrogénicos. Es de fundamental importancia realizar un buen diagnóstico de la vitalidad pulpar en concomitancia con el tratamiento ortodóncico, aun en dientes sin traumatismo previo, y con controles periódicos cada tres meses (Lau \& Wong). Dada la escasa referencia de la transiluminación para este fin (Fonseca), se propone su inclusión en los protocolos diagnósticos para su evaluación.

FONSECA, G. M. \& GUZMÁN, A. E. Orthodontic forces causing damage in pulpal condition. Report of two cases. Int. J. Odontostomat., 4(3):271-276, 2010.

ABSTRACT: The literature has suggested the implication of the use of orthodontic forces as producing mechanical damage, inflammatory reactions in the periodontium, periapical injury and root resorption. More commonly explored parameters in investigations of the tissue response to orthodontic forces consist of measuring the levels of pulpal oxygen and disturbances in dental pulp circulation. It has been demonstrated that the pulpal irrigation diminishes early when continuous applications of orthodontic forces are realized, even if these are of soft intensity, concluding that 
the magnitude of the forces must not necessarily be excessive to originate cell damage, which eventually might derive in signs such as simple crown discoloration to progressive obliteration or pulp necrosis. Two cases of orthodontically treated subjetcs with hemorrhagic extravasation and pulp necrosis (canine and lateral incisive respectively, both left upper) with absence of previous trauma are presented and the diagnostic protocols for the evaluation of the pulpal condition are discussed.

KEY WORDS: orthodontics, pulp injury, diagnostic procedures.

\section{REFERENCIAS BIBLIOGRÁFICAS}

Amir, F. A.; Gutmann, J. L. \& Witherspoon, D. E. Calcific metamorphosis: a challenge in endodontic diagnosis and treatment. Quintessence Int., 32:447-55, 2001.

Andreasen, F. M. \& Andreasen, J. O. Luxation injuries of permanent teeth: general findings. En: Andreasen, J. O.; Andreasen, F. M. \& Andersson, L. (eds). Textbook and color atlas of traumatic injuries to the teeth. $4^{\circ} \mathrm{Ed}$. Oxford, Blackwell Publishing, 2007.

Andreasen, J. O. \& Andreasen, F. M. Essentials of traumatic injuries to the teeth. $2^{\circ}$ Ed. Oxford, Blackwell Publishing, 2005.

Atack, N. E. The orthodontic implications of traumatized upper incisor teeth. Dent. Update, 26:432-7, 1999

Arwill, T.; Henschen, B. \& Sudvall-Hagland, I. The pulpal reaction in traumatized permanent incisors in children aged 9-18. Odontol. Tidskr.,75:130-47, 1967.

Bauss, O.; Röhling, J.; Meyer, K. \& Kiliaridis, S. Pulp vitality in teeth suffering trauma during orthodontic therapy. Angle Orthod., 79:166-71, 2009.

Bauss, O.; Schäfer, W.; Sadat-Khonsari, R. \& Knösel, M. Influence of Orthodontic Extrusion on Pulpal Vitality of Traumatized Maxillary Incisors. J. Endod., 36:203-7, 2010.

Brezniak, N. \& Wasserstein, A. Orthodontically Induced Inflammatory Root Resorption. Part II: The Clinical Aspects. Angle Orthod., 72:180-4, 2001.
Derringer, K. A.; Jaggers, D. C. \& Linden, R. W. A. Angiogenesis in Human Dental Pulp Following Orthodontic Tooth Movement. J. Dent. Res., 75:1761-6, 1996.

Dhopatkar, A. A.; Sloan, A. J.; Rock, W. P.; Cooper, P. R. \& Smith, A. J. British Orthodontic Society, Chapman Prize Winner 2003. A novel in vitro culture model to investigate the reaction of the dentinepulp complex to orthodontic force. J. Orthod., 32:122-32, 2005.

Drenker, E. Calculating continuous archwire forces. Angle Orthod., 58:59-70, 1988.

Fonseca, G. M. Diagnóstico del estado pulpar y sus complicaciones en dientes con historia de traumatismo. Tesis Doctoral, Facultad de Odontología, Universidad Nacional de Córdoba, Argentina, 2009.

Grossman, L. I. Práctica Endodóntica. $3^{\circ}$ Ed. Buenos Aires, Mundi, 1973.

Lau, P. Y. W. \& Wong, R. W. K. Risks and complications in orthodontic treatment. Hong Kong Dental Journal, 3:15-22, 2006.

McDonald, F. \& Pitt Ford, T. R. Blood flow changes in permanent maxillary canines during retraction. Eur. J. Orthod., 16:1-9, 1994.

Mostafa, Y. A.; Iskander, K. G. \& El-Mangoury, N. H. latrogenic pulpal reactions to orthodontic extrusion. Am. J. Orthod. Dentofacial Orthop., 99:30-4, 1991.

Nixon, C. E.; Saviano, J. A.; King, G. J. \& Keeling, S. D. Histomorphometric study of dental pulp during orthodontic tooth movement. J. Endod., 19:13-6, 1993.

Popp, T. W.; Artun, J. \& Linge, L. Pulpal response to orthodontic tooth movement in adolescents: a radiographic study. Am. J. Orthod. Dentofacial Orthop., 101:228-33, 1992.

Roberts, W. E. \& Ferguson, D. Cell kinetics of the periodontal ligament. En: Norton, L. A. \& Burstone, C. J. (eds). The biology of tooth movement. London, CRC Press, 1989.

Rotstein, I. Tooth discoloration and bleaching. En: Ingle J. I. \& Bakland, L. K. Endodontics. $5^{\circ} \mathrm{Ed}$. Hamilton, B. C. Becker, 2002. 
FONSECA, G. M. \& GUZMÁN, A. E. Fuerzas ortodóncicas como agentes vulnerantes de la salud pulpar. Reporte de dos casos. Int. J. Odontostomat., 4(3):271-276, 2010.

Santamaria, M. J. R.; Milagres, D.; Stuani, A. S.; Stuani, M. B. \& Ruellas, A. C. Initial changes in pulpal microvasculature during orthodontic tooth movement: a stereological study. Eur. J. Orthod., 28:217-20, 2006.

Spector, J. K.; Rothenhaus, B. \& Herman, R. I. Pulpal necrosis following orthodontic therapy. Report of two cases. N. Y. State Dent. J., 40:30-2, 1974.

Sulieman, M. An overview of tooth discoloration: extrinsic, intrinsic and internalized stains. Dent. Update, 32:463-4, 466-8, 471, 2005.

Woloshyn, H.; Artun, J.; Kennedy, D. \& Joondeph, D. Pulpal and periodontal reactions to orthodontic alignment of palatally impacted canines. Angle Orthod., 64:257-64, 1994.

Yamaguchi, M. \& Kasai, K. Inflammation in periodontal tissues in response to mechanical forces. Arch. Immunol. Ther. Exp., 53:388-98, 2005.
Dirección para correspondencia:

Dr. Gabriel M. Fonseca

Artigas 818, Dúplex A

Córdoba (5000)

ARGENTINA

Email: gabriel_fonseca@argentina.com

Received: 24-11-2010

Accepted: 08-12-2010 